\section{La educación terapéutica y su importancia en el ámbito de la dermatitis atópica}

\author{
Therapeutic education and its \\ relevance in atopic dermatitis
}

La Organización Mundial de la Salud define a la educación terapéutica como: "un proceso continuo de cuidados médicos, consistente en actividades educativas llevadas a cabo por los profesionales de la salud, creado para ayudar a los pacientes y a sus familiares a realizar su tratamiento y prevenir las complicaciones evitables mientras se mantiene o mejora la calidad de vida; incluye apoyo psicosocial, procedimientos y organización hospitalaria". Sus objetivos fundamentales son aportar información, proporcionar conocimientos prácticos para mejorar el cumplimiento del plan terapéutico $y$, sobre todo, para modificar el comportamiento.

Un aspecto esencial es que empodera a los pacientes para manejar su enfermedad mediante la adquisición de habilidades (autotratamiento, adaptación del tratamiento cuando sea necesario) a diferencia de simplemente proveer información. Se ha demostrado que la educación terapéutica contribuye eficazmente a prevenir complicaciones y a mejorar el cumplimiento del tratamiento, que aumenta la calidad de vida en numerosas enfermedades crónicas como la diabetes, el asma y las enfermedades cardiovasculares. ${ }^{1-3}$

La dermatitis atópica es la enfermedad inflamatoria crónica de la piel más común en la infancia, se caracteriza por una piel seca y con bajo umbral al prurito. La integridad de la barrera cutánea está alterada y eso facilita la pérdida transepidérmica de agua y la entrada de antígenos ambientales a la epidermis con la consecuente activación del sistema inmunitario que genera inflamación.

La dermatitis atópica tiene un impacto similar al de otras enfermedades crónicas en el bienestar y en la calidad de vida del paciente porque altera el sueño y la vida social y familiar. Su tratamiento es complejo pues incluye modificaciones en el estilo de vida, identificación constante de los factores predisponentes y el uso de agentes antiinflamatorios durante los brotes. ${ }^{1,4}$ No es raro que los pacientes tengan conceptos erróneos acerca de la enfermedad o de su tratamiento, eso los conduce a restar importancia al cumplimiento de su tratamiento y a la consecuente falla terapéutica. ${ }^{5}$ 
Las "escuelas de la atopia" han surgido en todo el mundo de forma independiente e incluyen a la educación terapéutica como una medida adicional en el tratamiento de la dermatitis atópica; pueden diferir en el tipo de intervención usada, los recursos humanos, las herramientas y las prácticas, pero tienen el objetivo común de elevar los índices de cumplimiento y, consecuentemente, disminuir la gravedad de la enfermedad y mejorar la calidad de vida de los pacientes. ${ }^{1}$

El consenso de expertos en dermatitis atópica (OPENED) analizó las diferentes estrategias de educación terapéutica utilizadas en cada una de 23 escuelas de atopia en 11 países. ${ }^{1}$ Existen estrategias, tanto individualizadas como colectivas, que consisten en sesiones multianuales de grupos grandes o pequeños en las que se educa a los pacientes y se comparten estrategias para tratar su enfermedad. El equipo es interprofesional e interdisciplinario, incluye médicos dermatólogos, pediatras, alergólogos, enfermeras, psicólogos y nutriólogos. Los pacientes reciben información general sobre la dermatitis atópica y recomendaciones para adquirir habilidades prácticas cómo prevenir brotes, utilizar el tratamiento y vivir con la enfermedad mediante diversas modalidades: académicas, interactivas, mediante juegos o canciones, utilizando material educativo o metáforas. A pesar de las diferencias institucionales y culturales de cada programa se recomienda que la educación terapéutica se integre al cuidado médico de los pacientes con dermatitis atópica porque: ${ }^{1}$

1. Su tratamiento es complejo y los pacientes y sus familiares son directamente responsables de aplicarlo y adaptarlo a las condiciones diarias de la enfermedad; por lo tanto, tienen que adquirir las habilidades necesarias para asesorar y adaptar el tratamiento y así controlar la enfermedad.

2. Beneficia a todos los pacientes y a sus familiares; especialmente aquéllos con historia de falla terapéutica, sin tratamiento eficiente o con poco apoyo social.

3. Permite la adquisición de conocimientos sobre la enfermedad: mecanismos, tratamientos y factores agravantes; habilidades prácticas (uso del tratamiento y su adaptación según la gravedad) y habilidades de relación (explicar la enfermedad a otros, saber a quién consultar y a quién pedir ayuda durante un brote grave). ${ }^{5}$

Los recursos pedagógicos que pueden utilizarse para enseñar las habilidades incluyen: técnicas de comunicación centradas en el paciente o familiar (escuchar activamente, empatizar, motivar); métodos educacionales diversos como presentaciones interactivas, mesas redondas, Iluvia de ideas, talleres, simulación de procedimientos, caracterizaciones, entrevistas y diversas herramientas dentro de las que se incluyen planes de acción escritos, carteles, libros con imágenes, videos y panfletos.

La evaluación de su eficacia es esencial en la educación terapéutica, puede realizarse mediante la medición de la gravedad de la dermatitis atópica con las escalas SCORAD o EASI, o con escalas que miden la calidad de vida del paciente. ${ }^{2}$ Rolinck-Werninghaus y su grupo ${ }^{6}$ demostraron que la educación terapéutica realizada por enfermeras especializadas aumenta la confianza de los padres para manejar el padecimiento y disminuye la gravedad y frecuencia de los síntomas. Por su parte, Mason y sus colegas ${ }^{7}$ encontraron que después de la educación terapéutica se incrementó el uso de emolientes, lo que redujo los síntomas de la dermatitis atópica sin incrementar los costos de atención.

Existen pocos estudios aleatorizados y controlados, que analicen el uso de la educación terapéutica en dermatitis atópica, que puedan ser evaluados objetivamente y comparados entre sí pues utilizan programas educacionales muy 
distintos y variables de desenlace diverso. Sin embargo en la revisión sistemática de Cochrane ${ }^{4}$ más reciente, en la que se incluyeron 10 estudios con un total de 2,003 participantes, se observó disminución de la gravedad de la enfermedad (usando SCORAD) y mejoría en la calidad de vida, especialmente cuando se realizaron sesiones de educación terapéutica mayores de 30 minutos y en niños menores de 7 años.

En nuestro país la educación terapéutica en dermatitis atópica se incorporó de manera formal en el 2012 tras la formación del capítulo mexicano de la Fundación para la Dermatitis Atópica, con su primera sede en el Instituto Nacional de Pediatría. En el 2014 se inauguró una segunda sede en el Instituto Dermatológico de Jalisco y, seguramente, continuarán aumentando dado el éxito de los talleres realizados hasta el momento.

Con la educación terapéutica se consolida el modelo de intervención que actualmente es utilizado por muchas escuelas para el tratamiento de la dermatitis atópica que consiste en: cuidados generales de la piel, prevención, tratamiento farmacológico y realización de talleres para pacientes y familiares (Figura 1). Hasta la fecha

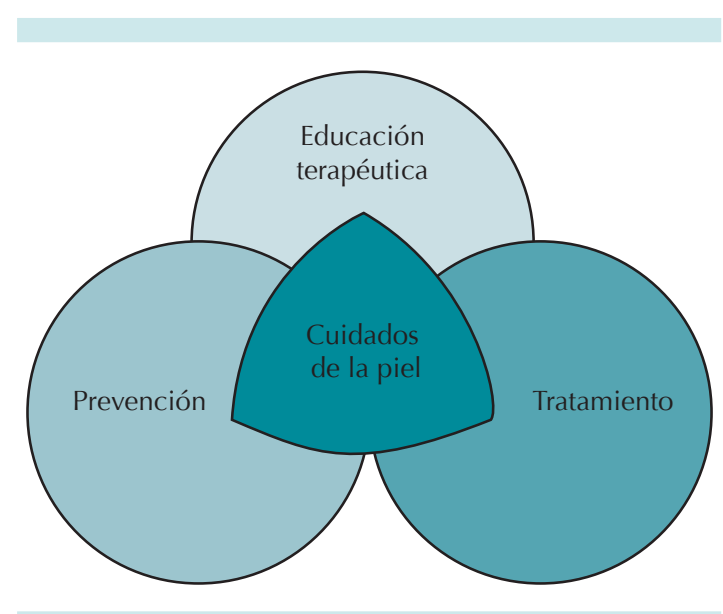

Figura 1. Modelo de intervención en dermatitis atópica que incluye los cuidados de la piel, la prevención, el tratamiento farmacológico y la educación terapéutica. se han incluido en los talleres 409 niños y sus familias; el siguiente paso será medir objetivamente la influencia de la educación terapéutica en la gravedad o en la cantidad de brotes de la dermatitis atópica.

\section{REFERENCIAS}

1. Stalder JF, Bernier C, Ball A, De Raeve L, Gieler U, Deleuran $M$, et al. Therapeutic patient education in atopic dermatitis: worldwide experiences. Pediatr Dermatol 2013;30(3):32934.

2. Barbarot S, Bernier C, Deleuran M, De Raeve L, Eichenfield $L$, El Hachem M, et al. Therapeutic patient education in children with atopic dermatitis: position paper on objectives and recommendations. Pediatr Dermatol 2013;30(2):199206.

3. Barbarot $\mathrm{S}$, Stalder JF. Therapeutic patient education in atopic eczema. Br J Dermatol 2014;170(Suppl 1):44-8.

4. Ersser SJ, Cowdell F, Latter S, Gardiner E, Flohr C, Thompson $A R$, et al. Psychological and educational interventions for atopic eczema in children. Cochrane Database Sys Rev. 2014;1:Cd004054.doi: 10.1002/14651858.CD004054. pub3.

5. Thompson DL, Thompson MJ. Knowledge, instruction and behavioural change: building a framework for effective eczema education in clinical practice. J Adv Nurs 2014;70(11):2483-94.

6. Rolinck-Werninghaus C, Trentmann M, Reich A, Lehmann C, Staab D. Improved management of childhood atopic dermatitis after individually tailored nurse consultations: A pilot study. Pediatr Allergy Immunol. 2015.doi: 10.1111/ pai.12338.

7. Mason JM, Carr J, Buckley C, Hewitt S, Berry P, Taylor J, et al. Improved emollient use reduces atopic eczema symptoms and is cost neutral in infants: before-and-after evaluation of a multifaceted educational support programme. BMC Dermatol 2013;13:7.doi: 10.1186/1471-5945-13-7.

\author{
Dra. Ma. Teresa García-Romero \\ Servicio de Dermatología \\ Instituto Nacional de Pediatría \\ teregarro@gmail.com \\ Dra. Marimar Sáez-de-Ocariz \\ Servicio de Dermatología \\ Instituto Nacional de Pediatría
}

